\title{
Percepción social sobre nanotecnologías en Cuba: Realidades y desafíos
}

\author{
Ariamnis Tomasa Alcazar Quiñones*
}

RESUMEN: El artículo aborda la percepción social de grupos sociales relevantes (GSR) que investigan el tema de las nanotecnologías en Cuba. A través de cada una de las dimensiones e indicadores del concepto de percepción social, se investiga el cuantioso mundo de conocimientos, de informaciones, de interacciones, de relaciones de cada grupo productor e investigador de esta tecnología en el país.

El estudio de la percepción social, de conjunto con varias técnicas y métodos del campo de estudios sociales de ciencia y tecnología (ESCT) permite encontrar un mapa gráfico de los principales colectivos de investigación del tema en el país así como de las principales etapas de investigación por las que atraviesa esta tecnología en el país.

Palabras Clave: Percepción social, nanotecnologías, Cuba.

ABSTRACT: The article discusses the social perception of relevant social groups (GSR) that investigate the topic of nanotechnology in Cuba. Through each of the dimensions and indicators of the concept of social perception, we investigate the large world of knowledge, information, interactions, and relationships of each group producer and researcher of this technology in the country.

The study of social perception, in conjunction with various techniques and methods from the field of Social Studies of Science and Technology (ESCT) to find a graphical map of the main groups of research on the subject in the country and the main stages of research by traversing this technology in the country.

KEYS wORDS: Social perception, nanotechnologies, Cuba

\section{INTRODUCCIÓN}

Uno de los rasgos distintivos que caracteriza al mundo contemporáneo es el impetuoso avance que la ciencia y la tecnología han adquirido. Todos los órdenes de la vida social, de una u otra manera, han sentido el impacto de este progreso. Según Núñez (1999) los poderes políticos y militares, la gestión empresarial, los medios de comunicación masiva descansan sobre pilares científicos y tecnológicos. Hasta la vida del ciudadano común está influida por los avances científicos y tecnológicos. Las nanotecnologías constituyen el paso más reciente alcanzado por la Revolución científico-técnica y prometen revolucionar la cosmovisión que se tiene del mundo.

Tradicionalmente, la tecnología ha sido entendida como mero factor productivo, desprovista de sus conexiones sociales. Esta visión aun persiste en nuestro país pues los adelantos tecnológicos no se han visto acompañados en todo momento de un pensamiento social que intente explicar el estado actual de las tecnologías que se aplican, así como sus impactos en la vida social (Pérez, 2008).

\footnotetext{
* MSc. por la Universidad de La Habana, Cuba. (aria@rect.uh.cu).
} 
El artículo ${ }^{1}$ pretende contribuir a la construcción de una visión integral de las nanotecnologías en Cuba, superando así la perspectiva tradicional y fragmentada. Es, además, una respuesta al reclamo de investigadores de la comunidad científica universitaria acerca de la necesidad de que ciencias como la sociología, la historia, entre otras, se interesen por el desarrollo de las ciencias naturales, exactas, por mencionar algunas.

\section{Algunos Antecedentes...}

Según Estévez (2009a: 6) “en Cuba las extremas dificultades económicas de la década de 1990, que perduran hasta hoy, contribuyeron al deterioro de la infraestructura científica del país". Ante esta situación, la nación se vio en la necesidad de enfocar sus esfuerzos hacia aquellos sectores que prometían un retorno a corto plazo de las inversiones, capacidad de auto sostenibilidad e impacto social masivo. Es así como se logró, por ejemplo, el desarrollo de la biotecnología en Cuba, la cual hasta nuestros días obtiene considerables progresos para el avance socioeconómico del país.

Sin embargo, durante el período antes mencionado, otras áreas del desarrollo científico sufrieron un retroceso considerable (Estévez, 2009a). Son algunas de estas áreas las que pudieran permitir el desarrollo de las nanotecnologías en el país, pues una de las aplicaciones de esta tecnología es la capacidad que posee de converger con otras ciencias. ${ }^{2}$ A pesar de esta difícil situación (Estévez, 2009a: 7) “Cuba ya puede hablar de una década de investigaciones en las tecnologías nano”. Según Barrere (2008) instituciones internacionales de vigilancia tecnológica, como el Observatorio Iberoamericano de Ciencia y Tecnología, reconocen no menos de doscientas noventa y siete publicaciones de Cuba en esta área desde el año 2000, lo que la ubica en el sexto lugar en el conjunto de países latinoamericanos y octava en países iberoamericanos.

Diferentes grupos de investigación trabajan el tema de las nanotecnologías en la actualidad en Cuba. El Ministerio de Educación Superior (MES), con todas las instituciones y centros de investigaciones adscritos a él, muestra considerables adelantos. No sólo por las publicaciones que realiza, sino por contar con especialistas reconocidos a nivel nacional e internacional en esta área. Además de estos logros, este ministerio es la única institución en Cuba que posee una red de nanotecnología con varios años de experiencia y que incluye a otras instituciones del Estado. (Estévez, 2009a: 8) La labor desempeñada por esta institución rebasa los marcos de los laboratorios de investigación de ciencias naturales, pues, durante casi veinte años, la Universidad de La Habana, junto a otras instituciones cubanas, viene promoviendo los estudios sociales de la ciencia y la tecnología (CTS). El interés por estos temas se vincula, de un lado, al intenso impacto (social, económico, político, cultural) que ejercen las ciencias y, la tecnología en la sociedad, así como la evidencia de sus profundos condicionamientos sociales, y, de otro, el énfasis colocado por la nación en el desarrollo científico y tecnológico y la necesidad de profundizar sus contribuciones sociales. ${ }^{3}$

\footnotetext{
1 El texto es un resumen de una investigación realizada en el año 2010 por la autora para la obtención de su título en licenciatura en sociología.

2 Aquí se refiere a la capacidad que tienen otras ciencias de conjugarse con las nanotecnologías para la formación de nuevos productos y resultados. Dentro de esta convergencia está la informática, la electrónica, la biotecnología, etcétera.

3 Esta información se obtuvo a partir de varias entrevistas exploratorias a diferentes miembros de esta cátedra. Su sitio web es <www.vriep.uh.cu/ctsuh>.
} 
El Ministerio de Ciencia, Tecnología y Medio Ambiente (CITMA) constituye otra de las instituciones que en el país despliega un esfuerzo por desarrollar las nanotecnologías (Estévez, 2009a). El Polo Científico ${ }^{4}$ ubicado en el oeste de la capital cubana cuenta con algunos centros de investigación que también desarrollan e investigan en materia de nanotecnologías. Resulta relevante destacar la existencia de otros actores sociales que trabajan el tema de las nanotecnologías desde diversas instituciones. Ellos son la Academia de Ciencias de Cuba (ACC), el Centro de Inspección y Control Ambiental (CICA), el Colegio de Ciencias Sociales de la Universidad de La Habana (UH) y periodistas de los medios de comunicación del país (Revista Bohemia, Juventud Técnica y periódico Juventud Rebelde).

Sin embargo, éstas y otras instituciones, consideradas grupos sociales relevantes (Bijker, 2008), tienen conocimiento sobre esta nueva tecnología desde una perspectiva estrecha. Según Estévez (2009b), poseen una visión instrumental de las nanotecnologías y las perciben como mero factor productivo.

Esta visión está condicionada por el enfoque tradicional con el cual se ha entendido la ciencia y la tecnología durante mucho tiempo. El mismo concibe a la ciencia sólo como un conocimiento probado, certero, como fuente de riqueza y bienestar social. Esta visión desconoce las conexiones sociales que tiene la ciencia, no sólo al interior de sí misma sino también con el contexto históricosocial en que se produce. Lo mismo ocurre con la tecnología, entendida como conocimiento aplicado, equipos, herramientas, éticamente neutral (Núñez, 1999). Según Estévez (2009a) el reto para Cuba en cuanto a nanotecnologías se presenta en dos direcciones interrelacionadas entre sí:

- Diseño de una estrategia efectiva de convergencia de la ciencia de los materiales, con la biotecnología, la informática y las ciencias cognitivas en la búsqueda de la sostenibilidad presente y futura de la industria cubana basada en la ciencia.

- Lograr, para esta convergencia, una estrategia efectiva que incorpore la dimensión sociológica y cultural del proceso como única vía de avanzar hacia una sociedad real del conocimiento.

El éxito de las nanotecnologías en Cuba no depende únicamente de la convergencia señalada y de la dimensión sociológica que ésta necesita sino también del apoyo del gobierno cubano para su aplicación y posterior difusión. A pesar de que en el país aún no se ha madurado y consensuado una estrategia para el desarrollo de las nanotecnologías (Estévez, 2009a) el gobierno cubano siempre le ha prestado especial atención al tema de la tecnología en el país. Ello se evidencia en el apoyo político e institucional (Núñez, Pérez y Montalvo, 2009) que se le ha dado a la biotecnología en el país, la cual cuenta con el apoyo y la iniciativa del líder cubano Fidel Castro.

\footnotetext{
${ }^{4}$ A partir de 2011, Cuba ha experimentado notables cambios en su política económica y social para perfeccionar el modelo socialista. Uno de estos cambios ha sido la integración del Polo Científico de biotecnología con instituciones farmacéuticas. En la actualidad BioCubaFarma es una empresa estatal con el objetivo de producir y comercializar medicamentos y servicios de alta tecnología para la salud de la población.
} 


\section{FUNDAMENTOS TEÓRICOMETODOLÓGICOS DE LA INVESTIGACIÓN:}

Los presupuestos teóricometodológicos empleados en la investigación provienen del campo de estudios sociales de ciencia y tecnología, ${ }^{5}$ en especial de la sociología de la tecnología.

A principios de los años 70 del siglo pasado se produce un movimiento, provocado en su origen por Thomas Kuhn, (Lamo de Espinosa et al., 1994) pero ampliado y sostenido por la crisis del estructural- funcionalismo y por el auge de las tradiciones fenomenológicas en la teoría sociológica, que produce una compleja metamorfosis en buena parte de la sociología de la ciencia que se genera a partir de este momento (Torres, 1994).

La sociología de la ciencia se encargó en su primer momento de analizar la ciencia como institución social, pero esta nueva interpretación se encarga de ver a la ciencia en acción y en torno a los procesos de estructuración del conjunto de las relaciones sociales científicas. ${ }^{6}$

En la década de los 80 comienza a desarrollarse el programa relativista, más conocido como el Programa Empírico del Relativismo (EPOR). Este programa, para el estudio que persigue, se traza tres etapas en las cuales declara sus proposiciones. La primera se refiere a la flexibilidad interpretativa de los resultados experimentales. Según Torres (1994) para este programa son muy importantes las diversas interpretaciones que se elaboran en torno a la ciencia y a sus investigaciones, por eso se entiende que los descubrimientos científicos pueden tener varias interpretaciones, cada una de las cuales hay que tenerlas en cuenta. La siguiente etapa apunta a los mecanismos de diversa índole que afectan la flexibilidad interpretativa señalada anteriormente. En este momento se consideran también el cierre de las controversias científicas al promoverse un consenso entre los investigadores. Por último, estas formas de cierre de las controversias científicas son analizadas en relación con el medio social y cultural en que se desarrollan dichas controversias. La cuestión de las controversias científicas considera que los hechos relevantes de la vida científica contemporánea no se dan tanto en los laboratorios, sino en otros espacios que se proporcionan en el marco científico.

Una interesante y fructífera extensión del EPOR es el programa SCOT (Social Construction of Science and Technology) desarrollado desde mediados de 1980 por Wiebe Bijker y sus colaboradores. En el SCOT se trata de estudiar empíricamente los artefactos y sistemas tecnológicos del mismo modo que el EPOR trata de abordar los produc-

\footnotetext{
5 Puede catalogarse con carácter interdisciplinar por concurrir en él materias como la filosofía y la historia de la ciencia y la tecnología, la sociología del conocimiento científico, la teoría de la educación y la economía del cambio técnico. (Cerezo, 1999; Bruun y Hukkinen, 2003) e incorporar a sus investigaciones los conocimientos tácitos. Propone conceptos integradores y transdisciplinares que den cuenta de la multiperspectiva y compleja realidad tecnológica actual. Es por ello que en su seno se generan conceptos y categorías analíticas para la comprensión de la tecnología como práctica social, artefactos, políticas, actores, valores, sistemas de gestión, patrones culturales etc. (Pacey, 1999; González, 2003, Thomas, 2008).

6 Posteriormente a la aparición del Programa Fuerte en la década de los años 70 del siglo XX, surgen a fines de este decenio otras orientaciones en torno a la nueva sociología del conocimiento. Estas orientaciones se constituyen en los estudios de vida de laboratorio, el programa relativista y de estudio de las controversias científicas, la etnometodología del trabajo científico, el análisis del discurso y la reflexividad y la teoría actor-red. Cada una de estas propuestas contienen diferentes enfoques de análisis para la tecnología.
} 
tos científicos. Se muestra la flexibilidad interpretativa y se analiza los mecanismos sociales mediante los que, en determinado contexto histórico y cultural se cierra tal flexibilidad y se consolidan las formas concretas de tecnología.

La metodología empleada en este programa consiste en la identificación de actores relevantes para el desarrollo de una tecnología dada, y de los problemas que estos actores se plantean en relación con la misma. Diferentes actores asociarán significados y problemáticas distintas a la misma tecnología, de tal modo que la evolución de su definición, la priorización de problemas a resolver y la estabilización de determinadas soluciones para los mismos dependerán del reparto de fuerzas y las estrategias negociadoras de los conjuntos de actores (González, 2003). Ejemplos clásicos los encontramos en el estudio del origen de la bicicleta, la baquelita y la bombilla fluorescente.

El concepto de grupos sociales ${ }^{7}$ relevantes (GSR) es uno de los conceptos clave dentro de la perspectiva constructivista en la sociología de la tecnología. Aunque fue en lo fundamental desarrollado por Pinch y Bijker (2008) su uso se ha extendido al resto de los estudios sociales de la tecnología.

Pinch y Bijker (2008) apuntan que este concepto es utilizado para denotar instituciones y organizaciones (como los militares o alguna compañía industrial específica), así como grupos de individuos organizados o desorganizados. El requerimiento clave es que todos los miembros de un determinado grupo social comparten el mismo conjunto de significados, vinculados a un artefacto específico. Al decidir qué grupos sociales son relevantes, primero debemos preguntar si el artefacto posee algún significado para los miembros del grupo social bajo investigación. Lo importante dentro de la sociología de la tecnología es que cada grupo representa una particular versión sobre un fenómeno determinado. En el caso de Pinch y Bijker se refieren a la bicicleta. Esta versión está en función de los intereses y necesidades de determinados GSRs en relación con la bicicleta.

Con el SCOT, el estudio social de la ciencia y el estudio social de la tecnología, que habían transitado por caminos independientes, comienzan a beneficiarse mutuamente.

Para Bijker (2008), la nueva sociología de la tecnología es "una excelente forma de comprender mejor cómo la tecnología es desarrollada, es decir, el proceso que lleva a lo que solemos llamar una invención, pero que no termina en eso, y nos permite entender por qué algunas tecnologías parecen funcionar en un sentido puramente técnico, pero luego fracasan cuando son injertas en la sociedad. 0 bien cuando una tecnología parece inferior, según la opinión de algunos expertos, y termina siendo exitosa". Este autor considera además, que la nueva sociología de la tecnología puede ampliar su agenda hacia las estandarizaciones que se realizan en las tecnologías

\footnotetext{
Este concepto tiene antecedentes en autores clásicos y contemporáneos de la sociología. Según Sutz (2003) la aproximación weberiana considera que el término relación social será usado para designar la situación en la cual dos o más personas se involucran en conductas; en las cuales cada una toma en cuenta significativamente el comportamiento de los otros, hecho que orienta dichas conductas. Según Giner (1998: 42) "un grupo está constituido por un número de individuos en una situación de mutua integración (aunque sea mínima), que sea relativamente duradera. El elemento numérico varía ampliamente, yendo desde los dos individuos hasta una vasta comunidad nacional. Estas colectividades son grupos en tanto sus miembros los son con un cierto grado de conciencia de pertenencia y, por ende, actúan de un modo que revela la existencia grupal; en otras palabras, su conducta sólo se explica enteramente si suponemos su pertinencia a esa estructura que llamamos grupo". Este autor considera que los miembros de los grupos deben tener cohesión en la interacción e integración mutua. Reconoce además, la dinámica de los grupos en tanto permite la interacción entre seres humanos y con las estructuras sociales.
} 
porque en definitiva "las estandarizaciones son tecnologías sociales que disciplinan y que empujan tanto a las personas como a las máquinas hacia un mismo marco conceptual" (Bijker, 2008).

Según Pinch (2008), la sociología de la tecnología sigue la trayectoria de los diferentes significados de una tecnología para poder observar cómo surgen nuevos significados y se desvanecen los antiguos, o cómo coinciden durante un periodo determinado.

Según Giner et. al. (1998), la sociología constructivista de la tecnología sostiene que los criterios científicos y técnicos no predominan en la configuración de las teorías y en la selección de las tecnologías. Existe un conjunto de soluciones para cada problema y los actores sociales intervienen en la elección final, mientras que la definición del problema cambia con frecuencia en el curso de la solución.

Estudios posteriores sobre la sociología de la tecnología consideran que esta perspectiva constructivista no le ha prestado atención a las consecuencias sociales (González, 2003) que pudiera tener la aplicación de una determinada tecnología, aunque Bijker (2008) ha reconocido la importancia de estos temas. Se evidencia una ingente preocupación por las decisiones que se deben tomar en torno al desarrollo tecnológico así como por los actores relevantes que deben tomar dichas decisiones.

Según Iañez y Sánchez (1998) se trataría, por tanto, de develar intereses y procesos sociales más profundos que pueden estar en la base de las elecciones sociales de la tecnología. Todas estas preocupaciones se dirigen no sólo a revelar la construcción social de los procesos tecnológicos sino también a la evaluación de dichos procesos. Con estas preocupaciones en relación con la tecnología, se introduce también una preocupación por los tomadores de decisiones en materia de tecnología. Quién establece las agendas políticas y con qué intereses lo hacen, son algunas de las interrogantes inferidas de estas investigaciones (Winner, 2000).

Estos análisis tienen como referente un concepto de tecnología no sólo artefactual sino además como un complejo que encierra políticas, valores, intereses, percepciones, etc. Por ello, Arnold Pacey sugiere que el fenómeno tecnológico sea estudiado, analizado, valorado, gestionado, en su conjunto, esto es como una práctica social, haciendo explícitos los valores que subyacen en la tecnología. Pacey (1990) aboga por una visión de tecnología no únicamente desde su dimensión instrumental sino, además, incluyente de los aspectos administrativos-organizativos, culturales etcétera:

- Dimensión técnica: conocimientos, capacidades, destrezas técnicas, instrumentos, herramientas y maquinarias, recursos humanos y materiales, materias primas, productos obtenidos, desechos y residuos.

- Dimensión organizativa: políticoadministrativa y gestión, aspectos de mercado, economía e industria, agentes sociales, cuestiones relacionadas con la actividad profesional productiva, la distribución de productos, usuarios y consumidores.

- Dimensión ideológicacultural: finalidades y objetivos, sistema de valores y códigos éticos, creencia en el progreso, etcétera.

Según Núñez (1999), la superación del enfoque estrictamente técnico permite una mejor definición del papel de los expertos. La tecnología, en tanto proceso social, debe tener en cuenta los factores que se relacionan con ella como la participación pública, las expectativas, las percepciones y juicios de los no expertos quienes también participarán en el proceso tecnológico. 
Para Estévez y Aragón (2008), la tecnología en apariencia neutral es en realidad, a través de su uso, portadora de una filosofía determinada, de relaciones de poder y condiciona en muchos casos la respuesta individual y colectiva de los fenómenos sociales.

Ante este enfoque que percibe la tecnología como proceso social permeado de valores, intereses, creencias sociales que pueden condicionar la visión social que se tenga de un fenómeno determinando; se hace imprescindible la investigación de los cientistas sociales en la "caja negra de la tecnología" así como de su grupo productor para desarrollar análisis e investigaciones, desde diversas aristas, que tributen a una mejor comprensión de los procesos tecnológicos. Esta visión de tecnología como proceso social es la que se asume en el presente artículo.

Dichas consideraciones permitirán el estudio de la percepción social de las nanotecnologías en Cuba en grupos sociales relevantes de la nación. La percepción social se emplea en la presente investigación como una herramienta que permitirá analizar los conocimientos, intereses, actitudes, significados de las nanotecnologías para los grupos sociales relevantes investigados.

La percepción es un concepto tratado desde la psicología, la filosofía y la sociología. Dentro de la sociología, la fenomenología le ha prestado gran interés a este término y a los significados. La sociología de la comunicación, con el análisis de los mass media, también ha considerado este término.

Resulta pertinente aclarar que en la bibliografía, los términos percepción, apropiación, cultura científica se emplean como sinónimos. Según López Cerezo (2010), la percepción es una etapa dentro de la cultura científica la cual puede abarcar otros procesos. Este autor entiende la cultura científica como un complejo multidimensional que integra elementos cognitivos, actitudinales, etc. Éste es un modo más amplio que incluye, además, disposiciones o inclinaciones al comportamiento.

Según Cerezo (2010), la cultura o la incorporación de la cultura científica, que podría ser apropiación, es un proceso analizable de un modo escalonado con escalones que interactúan entre sí. ${ }^{8}$

Una de las conceptualizaciones de percepción social según Cabo (2004) es la que sostiene que este término alude a una actividad que se estructura alrededor de los sentimientos, intenciones, motivaciones, valores, inserciones sociales y normativas que caracterizan al perceptor y al objeto percibido. La percepción social de ciencia y tecnología es el conocimiento o la idea que tiene una sociedad sobre ciencia y tecnología.

En el caso de esta investigación ese objeto percibido serán las nanotecnologías y el perceptor los actores que forman parte de los grupos sociales relevantes.

Cabo (2004) considera que la percepción social de ciencia y tecnología tendría su origen, más en el contexto concreto en el que ciencia y tecnología interaccionan con la sociedad, y en las significaciones sobre ciencia y tecnología, que en las características

\footnotetext{
8 El primer escalón sería la percepción la cual se refiere al interés por la ciencia, el segundo se refiere a la atribución de la importancia al desarrollo de la ciencia, una importancia que se puede expresar no solamente en términos de beneficios, sino también de perjuicios. Un tercer escalón será el del nivel de conocimientos de la ciencia, no sólo de los hechos sino también de conocimientos meta científicos, es decir, de conocimientos de las ciencias sociales acerca de la ciencia y la tecnología. Este escalón permitirá conocer los conflictos éticos y la postura de las ciencias sociales sobre el tema a investigar. Otro escalón alude a la asimilación de conocimientos científicos y que estos produzcan cambios en los sistemas de creencias de las personas y también en sus formas de comportarse. Este último es el de la apropiación pues es el de la incidencia de la ciencia en los sistemas de conducta y la vida de las personas.
} 
propias de la ciencia y tecnología en cuanto atributos objetivos que permiten discriminar lo que es o no científico.

La investigación se circunscribe al estudio de la percepción social de las nanotecnologías pues lo que pretende es la búsqueda de los significados, los conocimientos, valoraciones que realizan grupos de actores relevantes en el país con respecto a estas tecnologías en el contexto social cubano. Se reconoce la riqueza conceptual de la apropiación social, así como de sus más recientes aplicaciones en estudios empíricos. ${ }^{9}$

Los referentes empíricos en relación con la percepción social de las nanotecnologías en América Latina se ubican en Colombia, donde se han realizado estudios en un grupo de actores de ese país. Dicho estudio arrojó resultados muy relevantes y muy en concordancia con la lógica competitiva que se evidencia en la gran mayoría de los países latinoamericanos. Se percibe un gran conocimiento en el tema por parte de los estudiantes y profesores en áreas específicas, pero sobre los riesgos que pudiera generar dicha tecnología se evidencia una percepción difusa. En Cuba no se encuentra ninguna investigación acerca de la percepción social de esta tecnología.

Analizar la percepción social de estos grupos sociales relevantes en Cuba, con respecto a las nanotecnologías, permitirá representar circunstancias presentes y no presentes, anticipar situaciones venideras, recordar e interpretar sucesos, tejer narraciones, conservar y retransmitir discursos (Bueno, Santos, 2003) para poder ir configurando el destino y evolución de esta tecnología en el país.

Según Desdin (2010) la percepción social de las nanotecnologías en Cuba seguirá el mismo camino que la percepción social con la energía nuclear y los transgénicos. ${ }^{10}$ La percepción social se inclina en las etapas iniciales a los aspectos positivos que tiene una determinada tecnología. Asimismo (Desdin, 2010), cuando comienza a trabajarse con una tecnología todo el mundo ve los beneficios que pudiera brindar la misma. Sin embargo, cuando ocurren las catástrofes, los perjuicios, la tecnología es vista ya como algo totalmente perjudicial.

La opinión de Desdin (2010) es que eso es lo que ha pasado con la energía nuclear. Se ha ido a los dos extremos sin tener en cuenta los matices. Con las nanotecnologías ocurrirá algo similar, pues el foco de atención hoy está en los grandes beneficios de esta tecnología. Y las personas que conocen del tema están muy a favor de ella.

El análisis de la percepción social de los actores pertenecientes a los grupos sociales relevantes en cuanto al tema de las nanotecnologías permitirá conocer las bondades y desventajas de estas tecnologías para tener en cuenta los matices a los que se refiere Desdin (2010). Por ello, es pertinente la inclusión de las investigaciones de corte social en el desarrollo de las nanotecnologías en el país antes de que se atrinchere esta tecnología y sea difícil modificar la trayectoria de la misma en el país (Cerezo, 2010).

\footnotetext{
9 Uno de estos estudios empíricos es la cultura científica en Iberoamérica. Encuesta a grandes núcleos urbanos. Proyecto Estándar Iberoamericano de Indicadores de Percepción Pública y percepción ciudadana desarrollada por la organización de Estados Iberoamericanos (OEI).

${ }^{10}$ En el año 2007, se publicó un estudio en la revista Nucleus acerca de la percepción de la energía nuclear en la población de Ciudad de la Habana. Este estudio arrojó que el 89.5 \% de los encuestados acepta el uso de las técnicas nucleares por sus bondades y beneficios. Los medios de comunicación masiva son reconocidos como la mayor fuente de información de esta energía entre los encuestados. El 76.7 \% de los encuestados considera que la información sobre las técnicas nucleares en el país es insuficiente. Este autor considera, además, que todo descubrimiento científico en su génesis se considera la panacea a todos los problemas que enfrenta la realidad.
} 
Las herramientas metodológicas empleadas están sustentadas en la metodología cualitativa para la investigación social. ${ }^{11}$

El análisis cualitativo del comportamiento humano no se limita a la concepción positivista que considera que los hechos sociales ejercen una influencia externa y causal sobre el hombre, sino que también valora la importancia de cómo es vivida y percibida la realidad y su influencia en las ideas, sentimientos y motivaciones del hombre.

Se utiliza la entrevista en profundidad, el análisis de documentos y la técnica de bola de nieve ${ }^{12}$ para la consecución de los objetivos propuestos. Se emplea, además, un esquema sobre la trayectoria contexto/tecnológica que han tenido las nanotecnologías y la nanociencias en el país hasta el presente año. La entrevista a expertos se utiliza para indagar acerca del fenómeno en cuestión.

Para una mejor comprensión del objeto de análisis de la presente investigación resulta significativo conocer los términos técnicos y teóricos que emplean las nanotecnologías. Según Kreimer (1999), el desarrollo de metodologías sensibles es un requisito fundamental para los estudios empíricos de sociología de la ciencia. Dentro de estas metodologías sensibles uno de los problemas fundamentales es el de las competencias necesarias para internarse en el estudio de la práctica de los científicos (Knorr Cetina, 2005). Estas premisas de investigación permitieron a la investigadora participar en talleres, eventos y escuelas de verano. ${ }^{13}$ Dicha "formación" le permitió a la autora conocer los problemas condensados alrededor de los diferentes colectivos que trabajan el tema de las nanotecnologías en el país en la actualidad.

La muestra seleccionada para la realización de esta investigación la componen un grupo de actores pertenecientes a los grupos sociales relevantes identificados.

Los actores pertenecen al Centro de Ingeniería Genética y Biotecnología (CIGB), Centro Nacional de Investigaciones Científicas (CINC), Instituto de Ciencia y Tecnología de los Materiales (IMRE), Facultad de Química de la Universidad de la Habana, Centro de Aplicaciones Tecnológicas y Desarrollo Nuclear (CEADEN). La Academia de Ciencias de Cuba (ACC) y la Facultad de Filosofía e Historia de la UH. Dentro de los medios de comunicación masiva, la Revista Juventud Técnica, el periódico Juventud Rebelde, la revista Bohemia. El Consejo de Estado y el Partido de la Universidad de la Habana son otros espacios en los que se le ha prestado atención al tema que se investiga. El Centro de Inspección y Control Ambiental (CICA) es uno de los centros que investiga sobre el tema. Dos centros del Polo del Oeste de la Habana, CIGB y CNIC, cuentan con patentes en productos que incorporan nanotecnologías. Los especialistas de ambos centros de propiedad intelectual también forman parte de esta muestra.

Otra de las entrevistas realizadas para la investigación fue la que se le realizó al Dr. José Antonio López Cerezo, catedrático del Consejo Superior de Investigaciones

\footnotetext{
${ }^{11}$ Entre los antecedentes de esta propuesta se encuentran los estudios etnográficos, el interaccionismo simbólico con sus significados sociales y la fenomenología (Sandoval, 1996; Ritzer, 1993; Pérez, 2003).

${ }^{12}$ Herramienta de investigación que permite indagar acerca de las personas consideradas relevantes para un determinado campo de acción. En el transcurso de la investigación los entrevistados sugieren nombres, centros de investigación etc. los cuales llegan a repetirse en un momento dado. Aquí se cierra la muestra y se tiene la "bola de nieve"

${ }^{13}$ Entre los eventos en que se participó como asistente están: Congreso Internacional Universidad 2010, Conferencia Internacional de la Ciencia de los Materiales en la Era Nano, Seminario Taller "Impactos de la Nanociencias y la Nanotecnología: Retos y Perspectivas para América Latina: Llamado Único y en varios talleres y seminarios de investigación de la Cátedra CTS+I de la UH. Además, asistió al Curso Sociología de la Tecnología ofrecido por el Dr. Hernán Thomas de la Universidad Nacional de Quilmes, Argentina.
} 
Científicas (CSIC) y profesor de la Universidad de Oviedo en España, quien se dedica al tema de la percepción y apropiación social de la ciencia en Iberoamérica, a la MSc. Isarelis Pérez Ones de la Cátedra CTS+I de la Universidad de La Habana y al Dr. Julio César Casals Fernández de la Facultad de Psicología de la UH.

\section{Resultados}

Teniendo en cuenta las características del contexto históricosocial en que surgen y se desarrollan las nanotecnologías en Cuba, presentamos el análisis de su trayectoria en tres etapas. Dichas etapas pueden ser consideradas como una primera aproximación a una periodización. Es un recurso que se utiliza para facilitar la comprensión del objeto de estudio.

- Primera etapa: su inicio se ubica entre finales de la década de los años 80 e inicio de la de los 90 del siglo pasado y se extiende hasta 2005. Al interior de la etapa se pueden apreciar dos fases de desarrollo. La primera caracterizada por el derrumbe del socialismo europeo, el comienzo del "periodo especial" y la creación de grupos de investigación en las universidades cubanas. La segunda por el inicio de la investigación en nanotecnologías en diferentes grupos de investigación.

- Segunda etapa: se ubica su inicio en el año 2005 a partir de la propuesta de crear el Centro de Estudios Avanzados de Cuba (CEA). Lo consideramos como el primer esfuerzo de integración para unir los diferentes grupos de investigación que investigan el tema de las nanotecnologías y las nanociencias en el país. Creación de la Comisión ad-hoc.

- Tercera etapa: en el año 2009 comienza una nueva etapa. El desarrollo de la Conferencia Internacional "La Ciencia de los Materiales en la Era Nano" organizada por el IMRE. Este evento lo consideramos como el segundo esfuerzo de integración de grupos de investigación.

\section{Primera etaPA}

Esta etapa tiene dos fases fundamentales. La primera está relacionada con los elementos económicos, políticos y sociales que sirven de antecedente a la propia etapa y al proceso completo de surgimiento y desarrollo de las nanotecnologías en Cuba. La segunda fase responde a los comienzos mismos de la investigación en esta área en el país.

Primera fase. El derrumbe del campo socialista a partir de 1989 ocasionó graves problemas a la economía del país. En los años siguientes el PIB cayó en un 35\%, las exportaciones en un $85 \%$ y el suministro de combustibles en más del 75 \% (Núñez, Pérez, Montalvo; 2009). Estos disturbios afectaron directamente la actividad científica del país. Según Pérez (1997), a partir de 1990 comienza un proceso de transformación de la investigación científica en la Universidad de la Habana. La autora señala que luego del derrumbe del socialismo europeo y el cese casi total de los vínculos con la comunidad científica de estos países, ocurre un notable cambio en la reinserción de los grupos de investigación en el escenario científico internacional. En un periodo cor- 
to de dos o tres años estos grupos lograron restablecer sus vínculos orientados hacia América Latina, aunque de forma paralela fomentaron relaciones con países desarrollados. Se produjo un descenso del financiamiento para la investigación científica, universitaria, provocado por la situación económica que enfrentó el país desde inicios de la década de los años 90 del siglo XX el cual fue asimilado por los grupos de investigación, desarrollando diferentes estrategias para la gestión financiera.

Otra de las estrategias adoptadas por estos años estaba orientada hacia la solución de los problemas económicos del país. El principal móvil de desarrollo de esta estrategia era una preferencia por la investigación científica de corte más aplicado. Esta política incluía la actividad productiva como parte de la labor científica universitaria. La principal forma de adquisición de financiamiento en este caso era por vía de las empresas, ministerios que solicitaban determinada investigación a los grupos a través de la Universidad de La Habana (UH), Ministerio de Educación Superior (MES) y al Ministerio de Ciencia, Tecnología y Medio Ambiente (CITMA).

Según un entrevistado del GSR "comunidad de científicos de ciencias naturales" por estos años se desarrolla la base instrumental que acompaña al desarrollo actual de las nanotecnologías a nivel internacional. Por la situación precaria del país, así como de los grupos de investigación científica, Cuba se perdió esta revolución instrumental. Esta realidad condujo a que muchos grupos de investigación concentraron sus investigaciones en ciencias básicas.

Otra entrevistada de este GSR recuerda que como consecuencia del "periodo especial", los profesores de la Facultad de Física de la UH, tenían que arreglar sus propios instrumentos de trabajo. Muchos de los instrumentos que en la actualidad existe en la Facultad de Física, dice la entrevistada, datan de la década de los años 70. "No se han podido remplazar y cuando se rompen los arreglamos nosotros mismos". Esta entrevistada cuenta que cuando salía al extranjero tanto ella como sus compañeros de trabajo traían partes de instrumentos en sus equipajes.

Como resultado de este contexto de carencias económicas, ingenio científico y a través del contacto con el exterior es que se pudo realizar el microscopio de efecto túnel en Cuba. Ello fue posible, según la entrevistada, "por la tenacidad de un grupo de científicos cubanos en medio de tantas carencias y dificultades". Estos científicos son calificados por ella como "especies raras".

Antes de exponer el surgimiento de las nanociencias y de las nanotecnologías es necesario señalar que en el país, según refiere un entrevistado del GSR "comunidad de científicos de ciencias naturales", se habían realizado desde inicios de la década de los años 80 investigaciones enmarcadas en la escala nanométrica. Lo que pasa es que no se empleaban, señala este científico, los términos de nanociencias y nanotecnologías sino que se les conocía como física de baja dimensionalidad.

Segunda fase. En el año 2000, el Dr. Carlos Rodríguez ofrece una conferencia titulada "Materiales para el siglo XXI", con motivo de su ingreso como Académico de Mérito en la Academia de Ciencias de Cuba. Durante la preparación de la conferencia encuentra que acaba de salir la Iniciativa Americana y toda una conceptualización, un proyecto integrador en ese ámbito. La conferencia giró sobre el tema de las nanotecnologías, sobre la idea de que la frontera del conocimiento ahora estaba en los nanomateriales. Comenzaba así el interés por parte de científicos cubanos en esta área de investigación empleando ya los términos de nanociencia y nanotecnología.

De manera independiente, relata el entrevistado, comenzó a trabajar este tema Rolando Contreras, un médico del CNIC que trabaja en microbiología y que se intere- 
só por el tema desde el punto de vista de la biomedicina y de las aplicaciones médicas. Este científico comenzó a divulgar el tema dentro del Polo Científico del Oeste de la Habana. El interés por este científico y el CNIC de trabajar el tema de las nanotecnologías surge por una inspección del CITMA que obligaba a proyectar el desarrollo de este centro con vistas al año 2015, en los años 1999, 2000. Las soluciones encontradas estaban ligadas a la producción de las nanociencias y las nanotecnologías.

Según Rodríguez (2009), otra de las personas que impulsó el tema en el país por estos años fue Joaquín Tutor quien trabajaba en el Ministerio de Ciencia, Tecnología y Medio Ambiente (CITMA). Tutor creó a fines de 1990 la red CYTED que se llamó "Nanoestructuras Semiconductoras para la Electrónica y la Optoelectrónica". Se hacía énfasis en la parte de los semiconductores, pues en este tema el país tenía más experiencia desde años anteriores. Era ésta una de las pocas redes CITED que Cuba coordinaba y en la que participaban personalidades de otros países. Funcionó desde 1998 hasta el año 2003. Esta red realizó muchas actividades, publicaciones de libros, reuniones internacionales. Agrupaba a físicos en su mayoría.

Según Rodríguez (2009), el Instituto de Ciencia y Tecnología de Materiales (IMRE) comenzó a organizar talleres y escuelas de verano relacionadas con el tema a partir de 1999. Por esta época se presentó un punto en una reunión del Polo, que no tuvo trascendencia y en la cual hablaron del tema los actores antes mencionados. Para el año 2002 se creó en uno de estos talleres la Red de Nanotecnologías del Ministerio de Educación Superior con el apoyo de José Luis García Cuevas, director de Ciencia y Técnica de este ministerio. A la conformación de la Red asistieron Contreras por el CNIC, Ofelia Martínez por la CUJAE, Roberto Cao por la Facultad de Química, el Centro de Proteínas y Enzimas de la Facultad de Biología, el IMRE.

También la Universidad Central de Las Villas y más tarde el Dr. Villalonga de la Universidad de Matanzas.

Cuando comenzó la Batalla de Ideas en diciembre de 1999 hubo un proceso que se llamó "La ciencia y los científicos". En el marco de este proceso se realizó una reunión en la cual se planteó la necesidad de hacer prospectiva científica y tecnológica. Se mencionó y se abordó la importancia del estudio de las nanociencias y las nanotecnologías en este espacio. Esta propuesta fue asumida por el Observatorio Cubano de Ciencia y Tecnología en la persona de Soledad Díaz. Para el año 2002, se puso en circulación un documento titulado "Elementos para un análisis de las nanotecnologías en Cuba" dirigido a los niveles de dirección de primer nivel del país.

Hacia el año 2004 se consolidó el Programa Nacional de Nuevos Materiales y Materiales de Avanzada, e incorporó en su agenda la temática de las nanotecnologías, en la parte de materiales avanzados. Aunque se dieron algunos pasos, no se tomó la decisión a nivel de país de abrir un Programa Nacional de Ciencia y Tecnología (PNCT) en nanotecnologías. El Instituto de Ciencia y Tecnología de los Materiales (IMRE) promovió un taller con el Reino Unido para compartir ideas acerca del tema con especialistas ingleses, pero la idea de hacer un programa sin presupuesto no funcionó.

\section{Segunda etAPA}

Según Rodríguez (2009), en el año 2005, Fidel Castro Díaz-Balart plantea la idea de hacer un esfuerzo por desarrollar áreas de la ciencia a las que el país no había prestado la atención suficiente. Se pensaba desarrollar la bioinformática y las nanotecno- 
logías. A partir de ahí, señala Rodríguez (2009), se comenzó a trabajar en la idea de crear un Instituto, que no estuviera dentro del Polo Científico pero que le sirviera de retaguardia y como un lugar que se pudieran hacer investigaciones. La creación de este centro permitiría además el empleo del instrumental científico y tecnológico que nuestro país no conoció por la precaria situación económica que atravesó a inicios de la década de los años 90 del siglo pasado.

Se comenzó a elaborar una propuesta en la cual participó la Universidad de La Habana. Existía un pequeño comité integrado por Rolando Pérez, Óscar Rodríguez del INSTEC y Carlos Rodríguez del IMRE. Después se incorporó Fernando Guzmán, rector del INSTEC en aquel momento, y Ariel Felipe de Física, que actuaba como coordinador. Según Rodríguez (2009) se elaboró un documento que se le presentó a Fidel Castro, presidente del Consejo de Estado y de Ministros en aquel momento. La recomendación de Carlos Rodríguez, en torno a las áreas en que se pudieran desarrollar las nanociencias y las nanotecnologías, planteaba que era necesario acotar el Centro a aquellas áreas que interesaran más al país; las cuales serían áreas de desarrollo y de interés estratégico para la industria cubana y la seguridad nacional. Ejemplo de ello sería la biotecnología, la industria médicofarmacéutica, la defensa, la protección del medio ambiente y la producción de energía. Entre las líneas de investigación propuestas por este autor están los estudios de proteínas y otras moléculas, química supra molecular, nanomedicina, biología intracelular, nano toxicología, sistemas de liberación controlada de drogas, nano partículas para aplicación biomédica, interacción de nano objetos con organismos vivos, nano sensores, nano robots, diagnóstico y manipulación de células individuales. Considera este científico que este nuevo centro debería de estar abierto a la cooperación, que contara con un buen equipamiento analítico y otros materiales básicos; al que pudieran asistir científicos y proyectos relacionados con estas líneas de investigación y que serían aprobados por el consejo científico del centro. Podrían converger estudiantes de maestría y doctorado, profesores del país y del extranjero, un centro con una clara estrategia de generación y transferencia de propiedad intelectual y un staff relativamente pequeño.

Carlos Rodríguez, en el año 2007, le envía al rector de la Universidad de la Habana una nota acerca de este centro. Esta nota expone la concepción del plan inicial de este centro de investigación:

El Centro de Estudios Avanzados (CEA) es un centro de investigación adscrito al Consejo de Estado que se encuentra en construcción en el marco de la Batalla de Ideas. El primer edificio operativo sería para el 2008. La primera etapa de inversión concluiría en el 2010. Responde a la necesidad de fortalecer el campo emergente de las nanotecnologías, nanociencias, de las ciencias habidas, que son estratégicas para el futuro de la biotecnología y la industria medicofarmacéutica cubanas. El CEA se ha concebido como un centro abierto, con una plantilla relativamente reducida de físicos, químicos, ingenieros y especialistas que desarrollen proyectos conjuntos con profesores e investigadores del país y del extranjero dándole el máximo aprovechamiento a las capacidades experimentales que allí podrán encontrarse. El CEA en este sentido debe jugar el mismo papel que el CNIC en las décadas de los años 60, 70 y 80 del siglo pasado. Debe constituirse en un lugar para las investigaciones de ciencias básicas que se encuentran tan debilitadas de material en la actualidad. El proyecto científico del CEA se viene realizando con participación de especialistas de la UH, el Polo Científico, el INSTEC y el Consejo de Estado. En la actualidad se ha comenzado la creación de la cantera del CEA con la reserva de científicos de la universidad, estudiantes egresados de estas ciencias básicas, líderes científicos de otros centros de investigación. 
En la actualidad este centro aún se encuentra en fase constructiva, aunque en la parte de computación ya se están haciendo algunas cosas. Según un entrevistado del GSR "comunidad de ciencias naturales" se espera que este centro se encuentre funcionando completamente dentro de cinco años.

Este centro ha tenido muchas dificultades para la adquisición de los equipos de alta tecnología que se requieren para la investigación nanotecnológica. Según un entrevistado del GSR "tomadores de decisiones" ello se ha hecho evidente en la negativa de la empresa alemana Carl Zeiss de no venderle equipos al país para no poner en riesgo sus relaciones comerciales con el gobierno norteamericano.

Según un funcionario del GSR "tomadores de decisiones" se tiene la visión de crear una red del polo y una del CITMA para posteriormente enlazar todas estas redes junto a la del MES, única de su tipo en el país.

Según este entrevistado con el CEA se pretende elaborar plataformas tecnológicas que permitan desarrollar productos, aunque ello no signifique que no se le preste atención a la parte teórica, básica. Se proyecta extrapolar la experiencia del polo, no sólo desarrollar e investigar los conocimientos sino también la obtención de productos.

Se propuso además la creación en este periodo de la comisión ad hoc compuesta por diferentes especialistas quienes se han encargado de elaborar acciones estratégicas de seguridad de las nanotecnologías con respecto a la salud, la alimentación y el entorno. Esta comisión aun continúa trabajando en el tema. Está compuesta por especialistas del CITMA, del MES, y de salud pública, así como otros centros de investigación del país.

A pesar de estos esfuerzos, señala un entrevistado del GSR "tomadores de decisiones políticas, "actualmente se carece de una masa crítica de investigadores con el equipamiento y recursos necesarios para lograr una competitividad real a nivel internacional en nanotecnologías. Desde una perspectiva de la industria cubana, existe un casi completo desconocimiento de las implicaciones que a mediano plazo van a tener los desarrollos de las nanotecnologías".

Se financian hasta el presente un reducido número de proyectos de investigación en esta especialidad. No obstante, resulta evidente que el tránsito del saber básico a la aplicación industrial es a mediano y corto plazo según el comportamiento internacional y se requiere sentar las bases desde ahora para podernos insertar en esta Revolución tecnológica - considera este funcionario-.

Un análisis de esta etapa permite comprender la influencia del contexto social así como de otros factores en el cumplimiento de un fenómeno determinado. Las circunstancias políticas, económicas, sociales, etc., surgidas a partir del 2007, reconfiguran el plan inicial de este centro de investigación en el país.

\section{TERCERA ETAPA}

La Universidad de La Habana ha sido uno de los espacios que ha tratado el tema de las nanotecnologías y las nanociencias en el país. Según Rodríguez, en la actualidad, el MES cuenta con una red de nanotecnologías compuesta por la Universidad de la Habana (IMRE, BIOMAT, Facultad de Física y Facultad de Biología), Instituto Superior José Antonio Echevarría (ISPJAE), Centro de Investigaciones en Microelectrónica (CIME), Centro Nacional de Investigaciones Científicas (CNIC), Universidad Central de las Villas (UCLV), Centro de Bioactivos Químicos, el Centro de Ingeniería Genética y 
Biotecnología (CIGB), CEADEN, INSTEC, Centro de Química Farmacéutica (CQF), ICIMAF. Esta red tiene como objetivos impulsar la cooperación científica nacional e internacional en materia de nanociencias y nanotecnologías con vistas a mantener al país actualizado y desarrollar capacidades para la investigación científica y la formación de recursos humanos en este campo.

Promover especialmente la ejecución de proyectos de investigación, desarrollo e investigación tecnológica relacionados con las aplicaciones de las nanotecnologías a la industria biotecnológica y médicofarmacéutica cubanas.

El IMRE, un centro de investigación perteneciente a esta universidad, ha sido el centro que con más fuerza ha desarrollado estos temas hasta el presente. Según un entrevistado de la "comunidad de científicos naturales" ese interés se ha materializado en las escuelas de verano del año 2009 y en el Evento Internacional de noviembre del propio año que contó con la participación especial del premio Nobel de Química de 1996, Robert Curl. Significativo de este centro resulta el interés en torno a las implicaciones sociales, éticas, políticas y económicas de las nanotecnologías. Preocupación manifiesta en otros centros de investigación del país, pero que no se ha materializado en la realidad social.

Según Rodríguez (2009), el centro tiene varias fortalezas, entre ellas la formación de recursos humanos, una experiencia en esta materia de más de diez años, más de 200 artículos publicados, posicionamiento internacional, los profesores e investigadores de este centro son invitados a diferentes centros y universidades internacionales. Tienen acceso sistemático a facilidades multinacionales: European Syncroton Research Facility (Francia), Laboratorio Internacional de Luz Sincroton en Brasil, entre otras. El centro tiene, además, proyectos de colaboración con el CEA en la realización de nanomateriales para aplicaciones biomédicas y en la estructura y morfología de nanomateriales.

Resulta relevante destacar que la mayoría de los entrevistados reconoce la labor desplegada por este centro en materia de nanociencias y nanotecnologías. Ello se refleja en el análisis del sociograma (técnica de la psicología social que se emplea) donde el IMRE es el centro más reconocido por los colectivos de investigación, desde diversas áreas, que trabajan e investigan el tema de las nanociencias y las nanotecnologías. Este centro de investigación se preocupa además por realizar extensión universitaria de los conocimientos que poseen en estas tecnologías. Según un entrevistado del GSR "comunidad de científicos de ciencias naturales" y perteneciente al IMRE, esta institución tiene entre sus líneas de trabajo desarrollar esta actividad extensionista no sólo de las nanociencias y las nanotecnologías, sino también de otras líneas de investigación que ellos desarrollan. Los contactos de este centro rebasan los marcos de sus laboratorios y se encuentran extendidos en otros sectores de la población cubana.

\section{Grupos SOCIALES RELEVANTES IDENTIFICADOS}

(i) El GSR "comunidad científica de las ciencias sociales".

(ii) El GSR "comunidad científica de ciencias naturales". Este GSR tiene como problemática común hacer avanzar el conocimiento en el país en materia de nanociencias y nanotecnologías. Es común a este grupo, la cuestión de integrar aún más las disciplinas de las ciencias naturales para lograr de manera 
eficiente la convergencia que propugna el desarrollo actual de las nanociencias y las nanotecnologías.

(iii) El GSR “medios de comunicación masiva”. El grupo tiene en común difundir el tema de las nanotecnologías así como los avances que se van experimentando en el país en esta materia.

(iv) GSR “Organismos Regulatorios". Los entrevistados enmarcados en este grupo social relevante presentan una subdivisión; pues dos de ellos tienen en común la protección de la propiedad intelectual e industrial de las patentes en nanotecnología que se realizan en el país. Otras personalidades se dedican a investigar y estimular investigaciones acerca de los riesgos de las nanotecnologías en el medio ambiente para poder establecer regulaciones ambientales.

(v) GSR “Tomadores de Decisiones”. El significado que comparten es la pretensión de centralizar las decisiones en materia de nanociencias y nanotecnologías.

(vi) GSR "Extensionistas" (Activismo Social). Este grupo tiene como misión crear cultura científica en la población en relación con el tema.

(vii) GSR "Seguridad Nacional" Sabemos que tanto el Ministerio del Interior (MININT) como el Ministerio de las Fuerzas Armadas Revolucionarias (MINFAR) trabajan en el desarrollo de las nanotecnología; sin embargo, por cuestiones relacionadas con la seguridad nacional es imposible acceder a ellos.

(viii) GSR "Incógnito". La muestra no se cerró y se representa con un signo de interrogación para otros colectivos que trabajen el tema en el país y no hayan salido dentro de la técnica de bola de nieve.

El empleo del sociograma permite ver gráficamente las relaciones y vínculos que se establecen entre estos grupos sociales relevantes. Un análisis del esquema que se propone da cuenta de la acumulación de las relaciones entre varios centros de investigación (CNIC, IMRE, CIGB, Facultad de Química, Facultad de Biología, CEA). Coincidentemente son éstos los centros que más investigan en el tema de las nanociencias y las nanotecnologías en el país en la actualidad. El resto de los centros de investigación o se conectan entre ellos (CEADEN y CICA, por ejemplo) o no se relacionan con los centros de investigación que más investigan el tema.

La comunidad de las ciencias sociales no se encuentra relacionada con ninguno de los centros de investigación; ello evidencia la poca atención que estas especialidades le han prestado al tema de las nanotecnologías en el país. Los medios de comunicación constituyen otro de los GSR que conocen el estado del tema en el país e incluso se relacionan y tienen conocimiento acerca de lo que se investiga en el país en estas tecnologías. Ello se muestra en una serie de trabajos periodísticos desarrollados por varios espacios de divulgación realizados sobre el tema. La mayoría de los entrevistados de este GSR consideran que dichos trabajos se encuentran en una fase inicial, muy informativa, y con el objetivo de crear cultura científica en el país en materia de nanociencias y nanotecnologías. Ello se evidencia en el análisis de los artículos publicados por la revista Juventud Técnica en los cuales se aborda el estado actual del tema así como los logros que se han obtenido en algunos centros de investigación del país. ${ }^{14}$ Con toda esta labor desplegada por los medios, ningún GSR reconoce la parti-

\footnotetext{
${ }^{14}$ Esta revista ha publicado una serie de artículos relacionados con las nanotecnologías. Ellos son: "De nanociencias y otros micromundos", "Tras la quimera de oro", “Correteando entre átomos”. Éstos son los artículos a los que se alude en este análisis.
} 
cipación de ellos en materia de nanociencias y nanotecnologías. Ello permite corroborar la carencia de una visión social de las nanotecnologías entre los GSR analizados que integre a todos los sectores de la vida social. El tema de las nanotecnologías despierta miedos, temores e incertidumbres en la actualidad. Aún se desconocen a corto y mediano plazo los efectos que tendrán estas tecnologías en el entorno social y en la vida humana. Debido a las crecientes expectativas que se han generado en torno a estas tecnologías así como a sus potenciales aplicaciones, existen diversos sectores a los que no se pudo acceder. Aunque se conoce que se encuentran trabajando en el tema.

\section{Conocimientos, VAlores, PeRCEPCIONES....}

La mayoría de los entrevistados comparte un concepto común de nanociencias y nanotecnologías. Las nanociencias se refieren al estudio de las partículas en la escala nanométrica. Las nanotecnologías se refieren a la creación de productos teniendo como base la nanoescala. Un entrevistado de la comunidad de ciencias naturales considera que existen distintas comunidades científicas trabajando en esa escala, las cuales proponen producir una integración de ciencias distintas en esa escala. Integración, señala el entrevistado, "que por el momento es una potencialidad, no es una realidad."

Según este científico, el término nanotecnología es una construcción de la política científica, que refleja cosas objetivas, con el propósito de impulsar un grupo de ramas de la ciencia y de la tecnología y proponer posibles integraciones.

El asumir tanto uno como otro concepto no está en la dimensión técnica de los conceptos según diría Pacey, sino que está en el contexto en que se gestó este campo del conocimiento. Ello permite corroborar la tesis de que la tecnología es un proceso social (Núñez, 1999) y no puramente técnico como lo considera la visión tradicional de tecnología.

Ante la carencia de la infraestructura instrumental necesaria para el desarrollo de este campo, no quedó otra alternativa que centrarse en la investigación básica (Cao, 2010). Según un entrevistado perteneciente al GSR "Tomadores de decisiones políticas" lo que sí ha tenido un desarrollo notable en el país son las nanociencias. En lo esencial, "todas estas investigaciones han estado enmarcadas como nanociencias sin avanzar en desarrollos tecnológicos propios de las nanotecnologías. Es decir, artículos científicos, tesis de maestrías y doctorados con escasas patentes y nula transferencia tecnológica a la industria".

Sin embargo, a pesar de lo anterior, hoy en el país no se habla de nanociencias sino de nanotecnologías. La causa fundamental de esto está relacionada con el hecho de que el impacto real para el desarrollo del país hoy en día son las aplicaciones prácticas, o sea, las nanotecnologías (entrevistado del GSR “tomadores de decisiones políticas").

La nación cuenta con un personal calificado, con grandes conocimientos en investigaciones básicas en esta materia. Sin embargo, carece de la infraestructura tecnológica para poder aplicar dichas investigaciones básicas. Se percibe en el GSR "tomadores de decisiones políticas" una intención por incentivar en el país el desarrollo de nanotecnologías teniendo como base el alto nivel alcanzado en el país en materia de nanociencias.

Un entrevistado de este GSR considera que el país necesita aplicaciones prácticas, concretas en materia de nanotecnologías y los grupos de investigación que desarrollen esas aplicaciones prácticas deben reconocerse. Este entrevistado se cuestiona el 
otorgamiento de un premio a alguien que ha escrito veinte artículos y no a un grupo de investigación que desarrolló el microscopio de efecto túnel en Cuba.

En relación con esta intención de desarrollar productos en nanotecnología en el país, existen grupos de investigación que han logrado la creación de productos e instrumentos que incorporan y permiten el trabajo de esta tecnología. Ejemplo de ello ha sido el logro del interferón pegilado en el CIGB, el microscopio de efecto túnel logrado por un grupo de investigadores del IMRE, los endrímeros en el CNIC.

Los conocimientos en materia de nanociencias y nanotecnologías en su mayoría están permeados por la visión tradicional de tecnología. Sólo uno de los entrevistados, del GSR de la comunidad de ciencias sociales reconoce que "las nanotecnologías representan una forma contemporánea del conocimiento científico que tiene una problemática central de interés social y filosófico, es un área del conocimiento donde la ciencia se enfrenta al conocimiento no manejable, es decir, a una forma de producción de conocimiento científico donde la incertidumbre del conocimiento es lo estable, incertidumbre con respecto a las intervenciones, incertidumbre con respecto a las consecuencias, a las utilizaciones."

El reconocimiento de las nanotecnologías más allá de su dimensión técnica, artefactual, por parte de este autor, obedece a la formación que tiene este entrevistado en ciencias sociales y en el interés creciente en los adelantos e investigaciones que realiza el país en materia de biotecnología y nanotecnología.

La mayoría de los entrevistados de cada uno de estos grupos obtiene los conocimientos de nanotecnologías y nanociencias de Internet. Ésta es la principal fuente de información sobre el tema. Aunque algunos investigadores de la comunidad de científicos de ciencias naturales se han preocupado por difundir en la sociedad esta nueva tecnología, según una entrevistada de los medios de comunicación; esta acción no ha sido asumida por los otros grupos relevantes que se investigan. Los conocimientos sobre estas tecnologías en menor medida, según los entrevistados de la comunidad de científicos internacionales, provienen de los vínculos que tienen los centros de investigación con el exterior.

Algunos de los GSR (comunidad de ciencias naturales y tomadores de decisiones) tienen relaciones con distintos grupos de investigación provenientes del exterior. Ello se comprueba en el análisis de la matriz sociométrica. La cooperación internacional en materia de estas tecnologías en Cuba es muy amplia. El Observatorio Iberoamericano de Ciencia y Tecnología reconoce que en la región iberoamericana, Cuba es el país que más relación tiene con grupos de investigaciones foráneos.

El tema de las nanociencias y de las nanotecnologías despierta un gran interés en el país. Ello se evidencia en la preocupación por parte de los medios de comunicación de dar a conocer e informar sobre los avances que tiene Cuba en materia de esta tecnología. Según la mayoría de los entrevistados del GRS "medios de comunicación" los espacios en los medios para difundir este tema están muy debilitados y cuentan con poca participación en los principales medios de difusión masiva. Concuerdan estos entrevistados que su participación en la difusión de los adelantos científicos y tecnológicos en el país es prácticamente nula, pues sólo se cuenta con ellos para la celebración de los aniversarios de los centros de investigación

Esta tesis demuestra que los medios no son considerados por otros grupos sociales relevantes como para difundir estos conocimientos. Sin embargo, la totalidad de los entrevistados han realizado trabajos periodísticos relacionados con las nanotecnologías y se han encargado de difundir a la población los conocimientos que han 
adquirido. Ello se evidencia en el análisis de las publicaciones de la revista Juventud Técnica. Espacio que, según su directora, "no le dedica más atención al tema debido al poco espacio que tiene para hacerlo y el poco alcance que tiene esta revista."

La comunidad de ciencias sociales, en menor medida y no con la participación que debería tener, según un entrevistado de esta comunidad, no le presta gran atención al tema. Sólo científicos sociales aislados se preocupan por el tema.

Según un entrevistado del GSR "tomadores de decisiones", "hay grupos trabajando en el tema de las nanotecnologías en el país pero no hay unidad de esfuerzos, hay quienes quieren unir esfuerzo pero a su manera. Estamos trabajando en unir esos esfuerzos, estamos en muchas cosas a la vez, pero hoy ese tema no es estratégico para el país." Otra de las entrevistadas para la realización de esta investigación, perteneciente al GSR "medios de comunicación" considera que cuando se aborda el tema "da la sensación de la existencia de celos, cuestiones de búsqueda de reconocimiento entre los grupos que están trabajando en las nanociencias y las nanotecnologías, y eso, refiere la entrevistada, "puede despertar incomodidades."

El análisis de las opiniones, valoraciones, criterios de los entrevistados de los grupos sociales relevantes que se investigan, refleja la fragmentación de los grupos que trabajan el tema en el país. No se tiene aún una comunidad de esfuerzos en cuanto a nanociencias y nanotecnologías se refiere.

Según un entrevistado del GSR “tomadores de decisiones" las nanotecnologías se desarrollan en el país en función de las prioridades nacionales. Hoy es estratégico producir alimentos, esto no quiere decir que no le prestemos atención a este tema de las nanotecnologías. Según otro entrevistado del GSR "tomadores de decisiones" sí existe una voluntad política en materia de nanociencias y nanotecnologías. Este entrevistado cita las palabras textuales de Fidel Castro a mediados de 2006 cuando señala que "el CEA debía ser un centro ambicioso y de servicio a la humanidad". Señala este entrevistado y en consonancia con el entrevistado anterior, la necesidad de enmarcar ese centro en el contexto económico que vive el país.

La preocupación por el impacto que pudieran ejercer las nanotecnologías sobre el medio ambiente es otra de las líneas que más preocupa entre los investigadores del tema. Desde el punto de vista de las regulaciones ambientales no existe ninguna regulación en Cuba en materia de las nanotecnologías, ni siquiera en el mundo están muy difundidas estas regulaciones según un entrevistado del GSR "organismos regulatorios".

Este entrevistado considera que "en todo lo nuevo que surge siempre, la tecnología va delante, después los riesgos y problemas, y sólo después las regulaciones para controlar esos riesgos".

Las nanotecnologías están en una fase inicial pero a nivel de restricciones existen muy pocas. Según otro entrevistado del GSR "organismos regulatorios" la problemática en esta situación inicial de desarrollo de las nanotecnologías está en caer en una sobre regulación que puede ser más dañina que la misma carencia de regulación porque se pueden frenar futuros desarrollos.

El Ministerio de Ciencia, Tecnología y Medio Ambiente (CITMA) ha tomado algunas medidas para el país en relación con estas tecnologías. Hemos abogado, señala el director del centro de Control e Inspección Ambiental (CICA) por "realizar reuniones de colaboración con otros centros como el CEADEN en un proyecto de nano seguridad, por capacitarnos por aprender, y también por la colaboración de códigos de ética en el país relacionados con este tema por la carencia de regulaciones legales, de buscar normativas éticas, morales con la Academia de Ciencias de Cuba". 
En la actualidad en el país no puede haber una regulación en nano - considera este entrevistado-, pues sólo se puede pedir una licencia o una regulación a partir de que la ley lo disponga.

Un análisis de la Ley 81 del Medio Ambiente aprobada por la Asamblea Nacional del Poder Popular (ANPP), en 1997, establece la necesidad de contar con el CITMA para poder desarrollar algún proceso que se relacione con la tecnología o el medio ambiente. Esta ley es la principal ley ambiental en el sistema jurídico cubano. Su objetivo es establecer los principios que rigen la política ambiental y las normas básicas para regular la gestión ambiental del Estado, y las acciones de los ciudadanos y sociedad en general, a fin de proteger el medio ambiente y contribuir a alcanzar los objetivos de desarrollo sostenible del país. Esta ley no tiene en cuenta las nanotecnologías, pues data de 1997, y en el país no se hablaba con estos términos por esas fechas. El artículo 24 de dicha ley establece que

Toda actividad susceptible de producir efectos significativos sobre el medio ambiente o que requiera de un debido control a los efectos del cumplimiento de lo establecido por la legislación ambiental vigente, estará sujeta al otorgamiento de una licencia ambiental por el Ministerio de Ciencia, Tecnología y Medio Ambiente de conformidad con lo que al respecto estipule ese organismo, quien establecerá asimismo los tipos y modalidades de dicha licencia. El CITMA tiene la facultad de desarrollar resoluciones ministeriales para poder desarrollar algún proceso que ejerza influencia sobre el medio ambiente.

Este artículo le adjudica la potestad al CITMA de desarrollar resoluciones ministeriales para determinadas tecnologías. Esto se puede hacer si el CITMA decidiera desarrollar algo para las nanotecnologías. No se ha hecho aún posible por el poco conocimiento que hay en el tema y sobre el cómo después se van a controlar esas regulaciones. "Hace falta conocer los impactos ambientales que ejerce esta tecnología y cómo evitarlos para poder otorgar licencias", eso aún en Cuba no está claro, considera el director de este centro de control ambiental.

La realización de investigaciones sociales dentro de fenómenos tan complejos como la ciencia y la tecnología actuales resulta trascendental por el papel que dichos fenómenos ejercen en las sociedades modernas. Estas investigaciones permitirán poner de relieve los valores, fines, metas de los científicos y especialistas que realizan estos descubrimientos. Según una entrevistada del GSR "medios de comunicación" les corresponde a los sociólogos, a los investigadores de las ciencias sociales en general, encargarse de estos valores, fines, de los riesgos que pudiera generarse, evaluar además estos riesgos. Además, señala esta entrevistada, "tienen el compromiso de percibir y dar a conocer cuál es la mejor manera en que pueden hacerse llegar todos estos conocimientos a la población en general, para que no se identifique como algo dañino, en este caso la nanotecnología, cuando ésta no lo sea."

Otro entrevistado de este GSR considera que los grandes fracasos en Cuba de las nuevas tecnologías han sido no por el factor tecnológico sino por el factor cultural. Las ciencias sociales tienen un trabajo sumamente importante en esta readecuación entre tecnología y cultura. Las mentalidades tradicionales, de hábitos arraigados, a veces no permiten una correcta aplicación de las nuevas tecnologías.

Según este entrevistado, los países del primer mundo ya se han percatado de la necesidad de las ciencias sociales en el mundo nanotecnológico y, por ello, ya tienen personas trabajando en estos temas, en las dinámicas sociales, en las relaciones inter- 
personales, las regulaciones legales, patentes, etc. En Cuba lamentablemente no se ha comenzado en esta área como se debiera, señala este entrevistado.

Refiere este científico que en Cuba es necesario incluir a especialistas legales en el tema de las nanotecnologías por los peligros creados alrededor de estas tecnologías en el mundo capitalista. El centro al cual pertenece este entrevistado no cuenta con ninguna patente relacionada con estas tecnologías. Además, carecen de personal especializado para atender estos temas.

Otros centros de investigación pertenecientes al polo del oeste de la capital cubana cuentan con especialistas encargados de las patentes y de la propiedad intelectual. Estos centros (CNIC y CIGB) ya han desarrollado patentes en relación con las nanotecnologías.

\section{Conclusiones}

El estudio de la percepción social de las nanotecnologías así como la puesta en práctica de un conjunto de métodos y técnicas nos ha permitido el establecimiento de una trayectoria del desarrollo de las nanotecnologías en el país. Dicha trayectoria está condicionada por los fuertes cambios estructurales, sociales y políticos que ha padecido la nación cubana en las últimas décadas. Dicha percepción ha permitido conocer los criterios de un grupo de actores relevantes relacionados con el tema en cuanto a determinados tópicos referentes a las tecnologías nano. En la actualidad, el centro de atención en el país se concentra en las nanotecnologías, aun cuando éstas se hallan en un estado incipiente de desarrollo. Esto se debe, en lo fundamental, a la necesidad que tiene el país de potenciar las posibles aplicaciones industriales de estas tecnologías, por ejemplo, las asociadas con el sector de la industria biotecnológica y farmacéutica. Otro factor incidente en esta situación está relacionado con las fuentes de obtención y de legitimación de los conocimientos, así como con las fuentes de financiamiento. En ambos casos están asociadas a la cooperación internacional. La sociología, como ciencia reflexiva de la sociedad y de sí misma, debe explicar y comprender creencias, intenciones y significados si pretende explicar satisfactoriamente la vida social. La ciencia y la tecnología alcanzan espacios más preponderantes en nuestras sociedades actuales, dándole un sello distintivo al desarrollo social. Es por ello que este ensayo intenta responder algunas interrogantes en relación con el objeto de análisis que pretende; pero también puede realizar nuevos cuestionamientos a partir del espacio social investigado. ¿La fragmentación de la comunidad nanotecnológica es problema de esta tecnología en sí o del sistema de innovación tecnológica en general del país? ¿El problema es inherente a la tecnología o es producto de un sistema de relaciones sociales, del contexto social en que se investiga?

Resulta relevante cuestionar la división percibida entre las universidades y los centros de investigación del polo del oeste de la capital cubana. Un análisis del sociograma y de los resultados obtenidos permite comprender que son estos sectores quienes en la actualidad asumen los papeles más importantes en cuanto a nanotecnologías y nanociencias se refiere, pero en condiciones de estructuras muy diferentes. A pesar de la existencia de proyectos de investigación y procesos de formación entre ambos sectores, este estudio reconoce la fragilidad que tienen esas relaciones. Reconoce también la necesidad "de seguir trabajando en fomentar las interacciones sistémicas entre actores de la innovación con una perspectiva más intersectorial”. 
La historia parece repetirse una vez más, lo que cambia es el foco de atención, en este caso las nanotecnologías. Se reconocen las interacciones y relaciones entre los dos sectores anteriormente mencionados, sin embargo, el panorama muestra grandes limitaciones. El polo científico cuenta con estructuras que le permiten un mejor funcionamiento. Posee los recursos económicos y legales, entre otros, para poder desarrollar de manera más eficaz sus productos. La universidad, por su parte, realiza esfuerzos gigantescos para poder lograr un desarrollo en esta materia. No cuenta con recursos económicos ni legales para poder proteger sus invenciones fuera de las fronteras cubanas (Estévez, 2010). Estas conclusiones no sólo dan una panorámica sobre el tema en cuestión sino que abren la posibilidad a futuras investigaciones en el ramo, tan necesarias y pertinentes para nuestro país.

\section{REFERENCIAS BIBLIOGRÁFICAS}

Alvarado et al. (2007) "Actitudes de la población de Ciudad de La Habana hacia las técnicas nucleares". Nucleus, 42. Publicación semestral de la Agencia de energía nuclear y Tecnologías de Avanzada en Cuba. Ciudad de La Habana.

Barrere, Rodolfo et al. (2008) La nanotecnología en Iberoamérica. Situación actual y tendencias. Observatorio Cubano de Ciencia y Tecnología e Innovación. <http//www. oei.es/salactsi/nano.pdf>. Cátedra CTS+I de la Universidad de la Habana, <www. catedracts+i.vriep.uh.cu>.

Bijker, Wiebe y Pinch Trevor (2008) “La construcción social de hechos y de artefactos: o acerca de cómo la sociología de la ciencia y la sociología de la tecnología pueden beneficiarse mutuamente". En: Thomas, Hernán; Fressoli, Mariano y Lalouf, Bruno (comps.). Actos, actores y artefactos. Herramientas para el análisis de los procesos de cambio tecnológico y cambio social. Buenos Aires.

Bijker, Wiebe (2008) “La tecnología tiene que encajar en la sociedad”. Entrevista concedida por este autor durante la Primera Jornada Internacional de Estudios sobre Tecnología y Sociedad, organizada por el Instituto de Estudios sobre la Ciencia de la Universidad de Quilmes. <http://www.carlosianni.com.ar/blog/etiqueta/5/sociedad.html>, (diciembre, 2009).

Bueno, Carmen y Santos María Josefa (coords.) (2003) Nuevas tecnologías y cultura. Editorial Anthropos. Universidad Iberoamericana.

Cabo Hernández José M. y Enrique, Carmen (2004) Percepción social de la ciencia y la tecnología, tesis de maestría en CTS. Cuba: Universidad de Cienfuegos (disponible en formato digital).

Cao, Roberto (2010) Entrevista concedida a la autora (disponible en formato digital).

Casals Fernández, Julio César (2006) "Análisis del método sociométrico". En: Casals Fernández, Julio César (comp.). Conocimientos básicos de psicología social. La Habana: Editorial Félix Varela.

Desdin, Luis Felipe (2010) Entrevista concedida a la autora. Disponible en formato digital. Escobar, Alberto M. (2008) NBIC: Nano, Bio, Info, Cogno. La convergencia de tecnologías. Informe del Observatorio Cubano de Ciencia y Tecnología (OCCYT). La Habana: Academia de Ciencias de Cuba (disponible en formato digital).

Estévez Rams, Ernesto (2008) "Las nanotecnologías: buscando un enfoque integrador". Conferencia impartida por este autor en el IMRE. Universidad de La Habana. 
Estévez Rams, Ernesto y Aragón Fernández, Beatriz (2008) “Las nanotecnologías en un mundo disfuncional". Trabajo presentado en el evento Universidad 2010 (disponible en formato digital).

Estévez Rams, Ernesto (2009a) La fábula de los tres hermanos: las nanociencias y las nanotecnologías en el contexto cubano. Texto sin publicar.

Estévez Rams, Ernesto (2009b) Entrevistas concedidas por Ernesto Estévez, director del IMRE, mayo- junio del 2009.

Estévez Rams, Ernesto y Aragón Fernández, Beatriz (2009) Deshojando margaritas: ¿nanociencia o nanotecnología? (disponible en formato digital).

Estévez Rams, Ernesto (2010) Entrevista concedida por este científico cubano a la autora. Giner, Salvador (1998) Sociología. Barcelona: Ediciones Península.

Giner, Salvador, Lamo de Espinosa, Emilio y Torres, Cristóbal. (1998) Diccionario de Sociología. Madrid: Alianza Editorial.

González García, Marta I. (2003). Tema 5: Nuevas Aportaciones en los estudios sociales de ciencia y la tecnología. Curso Virtual. Escuela de Ciencia. Centro de Altos Estudios Universitarios OEI (disponible en formato digital).

Iañez Pareja, Enrique y Sánchez Cazorla, Jesús A. (1998) Una aproximación a los estudios de la sociología de la tecnología. <http://www.ugr.es/ eianez/Biotecnologia/cts.htm>, (2 de diciembre, 2009).

Knorr Cetina, Karin (2005) La fabricación del conocimiento. Un ensayo sobre el carácter constructivista y contextual de la ciencia. Buenos Aires: Universidad Nacional de Quilmes.

Kreimer, Pablo (1999) De probetas, de computadoras y ratones. La construcción de una mirada sociológica sobre la ciencia. Buenos Aires: Editorial Universidad Nacional de Quilmes.

Lamo de Espinosa et al. (1994) La sociología de la ciencia y del conocimiento científico. Madrid: Editorial Alianza Universal.

López Cerezo y Cámara Hurtado (2009) “Apropiación Social de la ciencia y participación ciudadana". En: Cultura científica en Iberoamérica. Encuesta a grandes núcleos urbanos. Proyecto estándar iberoamericano de indicadores de percepción pública y percepción ciudadana (2005-2009). Fundación Española para la Ciencia y la Tecnología. Organización de Estados Iberoamericanos. 85-105.

López Cerezo et al. (2002) Políticas del bosque. Madrid: Organización de Estados Iberoamericanos. Cambridge University Press.

López Cerezo, José Antonio (2010). Entrevista concedida a la autora en el marco del Congreso Internacional Universidad 2010. Palacio de Convenciones, La Habana, Cuba.

Núñez Jover, Jorge; Pérez Ones, Isarelis y Arriete Montalvo, Luis Félix. (2009) Biotecnología, universidad y política científica y tecnológica en Cuba: mirada a los avances y desafíos. La Habana.

Núñez, Jover Jorge (1999) La ciencia y la tecnología como procesos sociales. Lo que la educación científica no debería olvidar. La Habana: Editorial Félix Varela.

Pacey, Arnold (1990) La cultura de la tecnología como proceso social. México: Fondo de Cultura Económica.

Pérez Ones, Isarelis (1997) Aproximación al estudio de de las transformaciones actuales de la investigación científica en la Universidad de La Habana. Estudios de casos, tesis de licenciatura. Ciudad de La Habana. 
Pérez Ones, Isarelis (2008) Informe de oponencia a la tesis en opción al grado de licenciatura en sociología Análisis del programa de alfabetización informacional de Infomed: ¿Proceso tecnológico o proceso social?

Pérez La O, Julio (2003). Introducción al estudio de la Metodología de la Investigación Científica en las Ciencias Naturales. La Habana (disponible en formato digital).

Ritzer, George (1993). Teoría sociológica contemporánea. Primera y segunda parte. La Habana: Editorial Félix Varela.

Rodríguez, Carlos (2009). Entrevista concedida a la autora (disponible en formato digital). Sandoval Casilimas Carlos A (1996) Módulo cuatro. Investigación cualitativa. Instituto colombiano para el fomento de la Educación Superior. ICFES (disponible en formato digital).

Sutz, Judith (2003). Grupos de investigación en la Universidad de la República, Montevideo. Winner, Langdon (2000) Más allá de la innovación: ética y sociedad en una era de cambio incesante. Resumen de la conferencia 10 del Ciclo sobre Tecnología y Política, celebrado en la Universidad Internacional Menéndez Pelayo, octubre, Valencia. <http:// www.rpi.edu/>, (febrero, 2010).

\section{Anexo}

Mapa/Sociograma con los Grupos Sociales Relevantes identificados

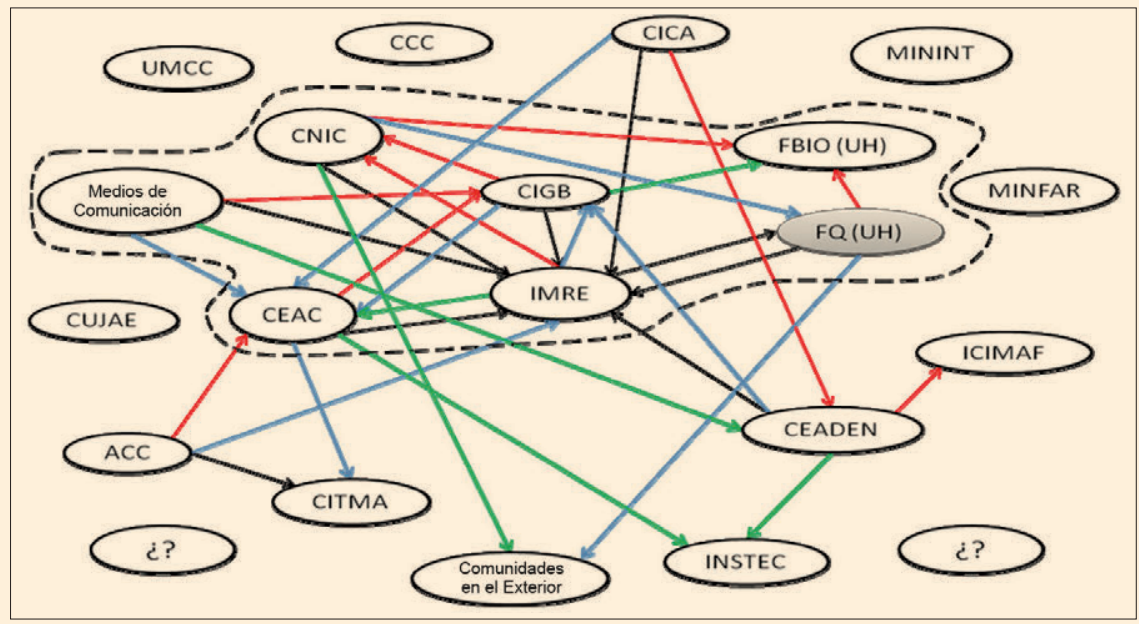

Leyenda:

Flecha negra: Primera elección.

Flecha azul: Segunda elección.

Flecha roja: Tercera elección.

Flecha verde: Cuarta elección. 